\title{
Transport inferred from consideration of particle orbits in drift turbulence
}

\author{
P M Bellan \\ California Institute of Technology, Pasadena, CA 91125, USA
}

Received 9 March 1992, in final form 19 October 1992

\begin{abstract}
Particle orbits in the 2D potential hills/valleys ('blobs') of low-frequency drift turbulence are analysed. For most of a given blob's lifetime a particle initially on the blob circulates on an orbit locked to that blob even though the blob shape and amplitude are changing. This locking corresponds to an adiabatic invariant being preserved. However, when the blob amplitude decreases to a critical value, the adiabatic invariant is destroyed, the locking ceases, and the particle detaches from the dying blob and attaches to a newly forming blob. This gives a detailed physical picture of the well known gyro-Bohm transport scaling $D \sim \gamma / k^{2}$ where $\gamma$ is the turbulence autocorrelation time (corresponding to blob lifetime) and $k$ is the perpendicular wavenumber (corresponding to blob diameter).

It is further shown that gyro-Bohm transport is consistent with global measurements in a wide variety of experiments, in addition to the large tokamaks to which it has traditionally been applied. Finally, it is shown that ion orbit behaviour becomes qualitatively different if the blobs become sufficiently sharp and steep; in particular, ions develop stochastic trajectories if located on $2 D$ potential hills (diameter $\pi / k_{\downarrow}$, amplitude $\bar{\phi}$ ) satisfying $\left(k_{\perp} \rho_{\mathrm{s}}\right)^{2} e \bar{\phi} / T_{\mathrm{c}}>0.3$ where $\rho_{s}=\left(\kappa T_{\mathrm{c}} / m_{\mathrm{i}}\right)^{1 / 2} / \omega_{\mathrm{cl}}$.
\end{abstract}

\section{Introduction}

The low-frequency elastrostatic drift wave turbulence ubiquitously observed in magnetically confined plasma (Liewer 1985 Wooton et al 1990) is suspected to cause observed energy and particle transport because

(i) empirically determined energy transport scaling laws (Christiansen et al 1990) have the gyro-Bohm scaling predicted by fluid drift transport theories (Waltz et al 1990);

(ii) observations (Ritz et al 1990) of the H-mode correlate improved confinement with reduction in edge drift wave turbulence; and

(iii) helium transport measurements (Synakowski et al 1990) are consistent with low-frequency elastrostatic turbulent transport.

Surprisingly, suprathermal (i.e. MeV) ions have been observed (Zweben et al 1990) to have neo-classical confinement (i.e. much better than gyro-Bohm scaling).

We discuss here a particle orbit view of drift turbulence transport. This gives the usual gyro-Bohm transport scaling, but provides additional insight into how individual particles are actually moving. The gyro-Bohm transport scaling is compared to various experiments and it is shown that, besides the established result that gyro-Bohm gives reasonable scaling for large tokamak experiments, gyroBohm 
also gives good predictions (or at least an upper bound) for a wide variety of non-tokamak experiments and also small tokamaks. In the extreme limit of very high amplitude turbulence (such as may be found in edge plasmas), it is shown that a stochastic heating of ions results.

\section{Review of observed properties of drift turbulence}

Observed edge drift turbulence is broad band, with $\omega \ll \omega_{\text {ci }}$, and has short correlation length perpendicular to $\boldsymbol{B}$ and long correlation length parallel to $\boldsymbol{B}$. The typical (Wooton et al 1990) observed perpendicular wavenumber $k_{\perp}$ is such that $k_{\perp} \rho_{\mathrm{s}} \approx 0.1-0.3$ where $\rho_{\mathrm{s}}=\left(k T_{\mathrm{s}} / m_{\mathrm{j}}\right)^{1 / 2} / \omega_{\mathrm{c} 1}$, while the measured (Ritz et al 1988) parallel wavenumber $k_{\|}$is such that $\omega / k_{\|} u_{T_{\mathrm{c}}} \approx 0.3-1$. All modes of the turbulence convect at approximately the same phase velocity $u_{\mathrm{ph}}^{\mathrm{pl}}$ as measured in the plasma frame, and this phase velocity is (Zweben and Gould 1985, Wooton et al 1990) in the direction $\nabla n \times B$ with a magnitude $u_{\mathrm{ph}}^{\mathrm{pl}} \approx u_{\mathrm{D}}$ where $u_{\mathrm{D}}=T_{\mathrm{e}} / e B L$ is the electron diamagnetic drift velocity and $L$ is the density gradient scale length; i.e. the turbulence lies in the drift wave regime. Because of plasma $\boldsymbol{E} \times \boldsymbol{B}$ rotation associated with a DC radial field that typically exists in the plasma, the phase velocity observed in the laboratory frame is $u_{\mathrm{ph}}^{\mathrm{lab}}=u_{\mathrm{ph}}^{\mathrm{pl}}+B^{-1} \partial \phi_{\mathrm{DC}} / \partial x$, where $\phi_{\mathrm{DC}}$ is the DC plasma potential and $x$ is in the minor (i.e. $-\nabla n$ ) direction. The turbulence amplitude scales (Liewer 1985, Wooton et al 1990) as $\bar{n} / n \approx\left(k_{\perp} L\right)^{-1}$; in the interior $\left(k_{\perp} L\right)^{-1} \ll 1$, but at the edge $\left(k_{\perp} L\right)^{-1} \sim 0.2-0.5$. Edge probe measurements (Wooten et al, 1990) show that the potential fluctuations $\tilde{\phi}$ scale as $e \tilde{\phi} / T_{\mathrm{c}} \approx 0.5-0.8$, and that $e \tilde{\phi} / T_{c}>\tilde{n} / n$ (i.e. non-Boltzmann behaviour). Hence, to the extent probes can be inserted, it is observed that

$$
\left(k_{\perp} L\right)^{-1}<e \tilde{\phi} / T_{\mathrm{e}} \text {. }
$$

The non-Boltzmann behaviour, the experimentally observed lack of dependence on collisionality (Wooton et al 1990, Zweben and Gould 1983), and the experimental observation that $\omega \sim k_{\|} u_{T_{\mathrm{c}}}$ combine to suggest that in the edge plasma the drift turbulence is driven unstable by Landau damping (which peaks when $\omega \approx k_{\|} u_{T_{\mathrm{c}}}$ and which is independent of collisions).

Two-dimensional probe measurements (Zweben and Gould 1985, Zweben 1985) show that the edge turbulence consists of random hills and valleys or 'blobs' of potential and density with transverse dimension $\sim \pi / k_{\perp}$. In Zweben (1985) blob lifetimes were observed to be $\sim 10 \mu \mathrm{s}$, the characteristic blob velocity was $2 \times$ $10^{5} \mathrm{~cm} \mathrm{~s}^{-1}$ and the characteristic blob diameter was $1 \mathrm{~cm}$. Thus, for Zweben (1985) at least, a typical blob travelled about twice its diameter during its lifetime. We assume that this lifetime is typical and so characterize the typical blob lifetime as being the time it takes a blob to convect twice its diameter, i.e. $\tau_{\text {life }} \sim h \pi / k_{\perp} u_{\mathrm{D}} \approx$ $h \pi / \omega^{*}$ where $h \approx 2$. We also assume that the interior turbulence is qualitatively similar to the edge turbulence, differing only in amplitude, as given by the $\tilde{n} / n \approx\left(k_{\perp} L\right)^{-1}$ scaling. Our justification is that interior and edge fluctuations as observed by scattering are essentially similar (Mazzucato 1982) except for amplitude and Doppler shift of the frequency due to plasma rotation.

\section{Condition for blobs to exist in the wave frame}

We begin our analysis by representing these interior, convecting blobs by the $2 \mathrm{D}$ electrostatic laboratory frame fluctuating potential $\bar{\phi}\left(x, y-u_{\mathrm{ph}}^{\mathrm{lab}} t, t\right)$ where $y$ is in the 
$\nabla n \times B$ direction (electron diamagnetic drift), $x$ is in the $\nabla n$ direction, and $B=B \hat{z}$. The second $t$ in the argument of $\tilde{\phi}$ represents absolute variation in time. Thus the total potential as seen in the laboratory frame is

$$
\phi=\tilde{\phi}\left(x, y-u_{\mathrm{ph}}^{\mathrm{lab}} t, t\right)+\phi_{\mathrm{DC}}(x) .
$$

To understand absolute versus convective time dependence in equation (2) we first suppress the absolute time dependence in $\bar{\phi}$ and consider ion motion in the purely convective $2 \mathrm{D}$ turbulent potential,

$$
\phi=\bar{\phi}\left(x, y-u_{\mathrm{ph}}^{\mathrm{lab}} t\right)+\phi_{\mathrm{DC}}(x)
$$

(electrons, for which parallel motion may be important, will be considered later). On changing to the wave frame (which has coordinates $x^{\prime}=x, y^{\prime}=y-u_{\mathrm{ph}}^{\mathrm{iab}} t$, $\left.u_{x}^{\prime}=u_{x}, u_{y}^{\prime}=u_{y}-u_{\mathrm{ph}}^{\mathrm{lab}}\right)$ the wave-frame Lorentz equation is found to be $m \mathrm{~d} u^{\prime} / \mathrm{d} t=$ $q\left[-\nabla \phi^{\prime}\left(x^{\prime}, y^{\prime}\right)+u^{\prime} \times B\right]$ where

$$
\phi^{\prime}\left(x^{\prime}, y^{\prime}\right)=\tilde{\phi}\left(x^{\prime}, y^{\prime}\right)-u_{\mathrm{ph}}^{\mathrm{pl}} B x^{\prime} .
$$

We now ask whether blobs exist in the wave frame. Equation (4) shows that the conditions for blobs to exist in the wave frame are (i) that $\bar{\phi}$ should consist of blobs and (ii) $\max \left(k_{\perp} \tilde{\phi}\right)>u_{\mathrm{ph}}^{\mathrm{pl}} B$; since if condition (ii) were not fulfilled, the second RHS term of equation (4) would annihilate the localized maxima of the blobs. Since $\tilde{\phi}$ does indeed consist of blobs and, using $u_{\mathrm{ph}}^{\mathrm{pl}} \approx u_{\mathrm{D}}$ together with the 'non-Boltzmann' condition implicit in equation (1), we see that the second term on the RHS of equation (4) is indeed insufficient to annihilate the localized 2D blob extrema. Thus $\phi^{\prime}$ also consists of blobs. It is important to realize that the effects of the poloidal $\boldsymbol{E} \times \boldsymbol{B}$ rotation caused by the DC radial electric field cancel when evaluating the RHS of equation (4) so that all that counts is $u_{\mathrm{ph}}^{\mathrm{pl}}$, not $u_{\mathrm{ph}}^{\mathrm{lab}}$ which is often much larger than $u_{\mathrm{ph}}^{\mathrm{pl}}$ and sometimes of opposite sign.

At this point it is worthwhile noting that these blobs appear to be examples (in the electrostatic limit) of the solitary vortices described by Liu and Horton (1986). The difference between their analysis and ours is that Liu and Horton have postulated that vortices exist, and then developed a self-consistent, nonlinear, two-fluid description. Here, we have simply noted from experimental measurements that blobs do exist in the laboratory frame, and have then deduced the condition for blobs to exist in the wave frame (where the blobs now give rise to vortex motion). Our analysis is complementary to Liu and Horton's-while being much less rigorous mathematically, it is more intuitive since we focus our attention on the properties of particle orbits in the vortex, rather than on the rather complicated, self-consistent, fluid description of the vortex.

\section{Orbits in purely convecting blobs}

The advantage gained in moving to the wave frame is that $\phi^{\prime}$ is time independent. It also has a slight slope in the $x$ direction; however, this slight slope is of no great consequence to a specific blob. McChesney et al (1987) showed that when $\alpha=m k_{\perp}^{2} \phi / q B^{2}>1$, guiding centre theory breaks down and ions develop stochastic orbits. We now assume $\alpha \ll 1$ so the orbits are not stochastic, but will return to stochasticity later. Since $\phi^{\prime}$ is time-independent, the wave-frame guiding centre 


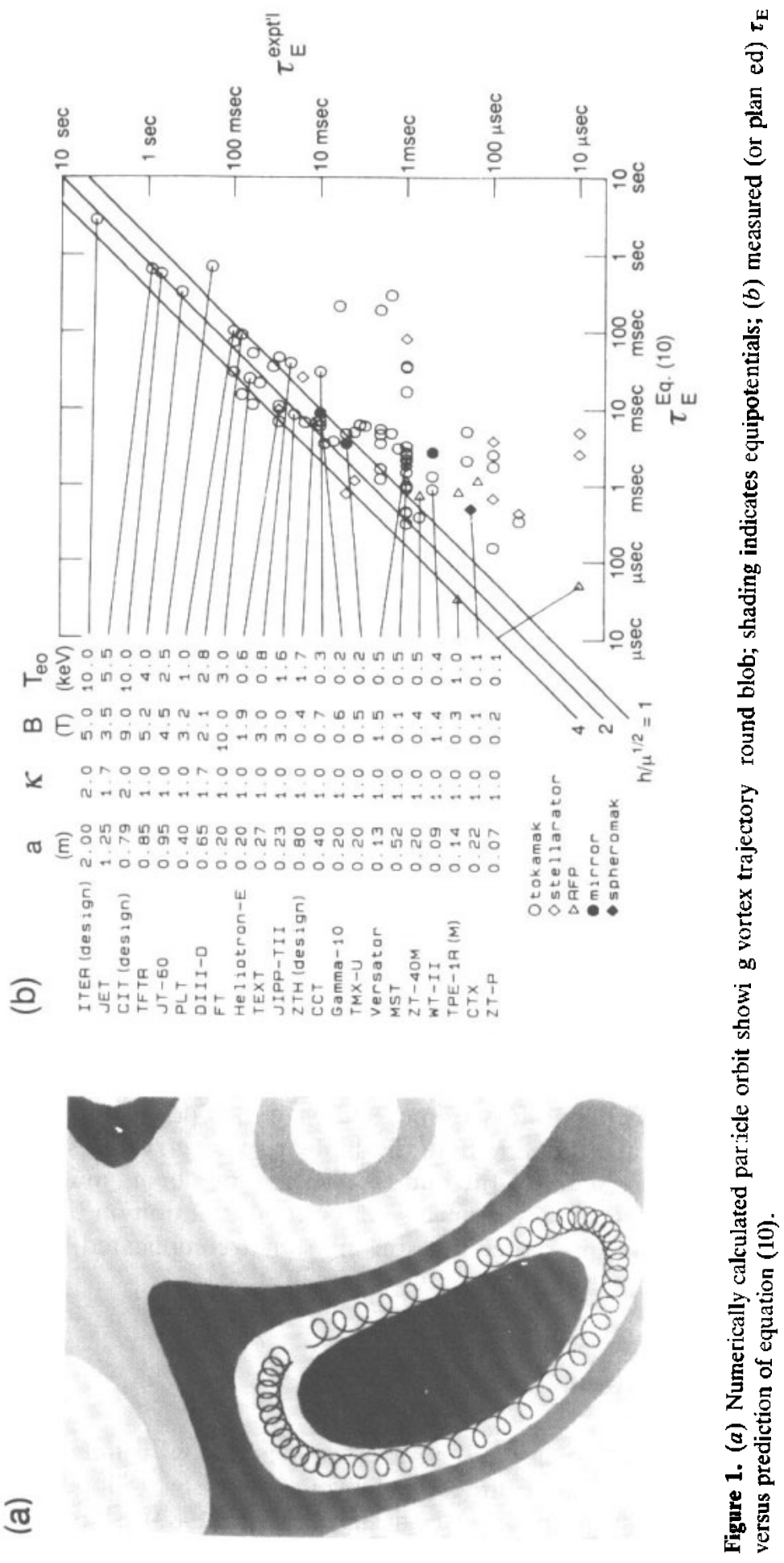


motion is given by $\boldsymbol{u}^{\prime}=\boldsymbol{u}_{\mathrm{E}}+\boldsymbol{u}_{\mathrm{P}}$ where $\boldsymbol{u}_{\mathrm{E}}=-\left(\nabla \phi^{\prime} \times \boldsymbol{B}\right) / B^{2}, \boldsymbol{u}_{\mathrm{P}}=\left(m / q B^{2}\right)(\mathrm{d} / \mathrm{d} t)$ $\left(-\nabla \phi^{\prime}\right)$. Because $\alpha \ll 1$ and $\omega \ll \omega_{c i}$, the polarization drift $\boldsymbol{u}_{\mathrm{P}}$ is much smaller than the $E \times B$ drift $u_{\mathrm{E}}$. Since $u_{\mathrm{E}} \cdot \nabla \phi^{\prime}=0$ the ion repeatedly circumnavigates the blob following the equipotential $\phi^{\prime}\left(x^{\prime}, y^{\prime}\right)=$ constant (cf figure $1(a)$ ); i.e, the ion is frozen to a blob. Consideration of the polarization drift shows that there will be a slight deviation of this vortex flow from the equipotentials, but the mean deviation over one circumnavigation vanishes because $\oint \boldsymbol{u}_{\mathrm{p}} \mathrm{d} t=-\left(m / q B^{2}\right) \oint \mathrm{d} t \mathrm{~d}\left(\boldsymbol{\nabla} \phi^{\prime}\right) / \mathrm{d} t=0$. This description of particle motion is similar to Horton (1990), but additionally includes polarization drift effects and also the consideration of DC electric fields.

The vortex trajectory, $\phi^{\prime}\left(x^{\prime}, y^{\prime}\right)=$ constant, is highly nonlinear in $\phi^{\prime}$. All ions are trapped in their respective blobs, and the motion is very different from what would be calculated from linear theory. When using linear theory, one assumes that the ion motion is a perturbation of the unperturbed orbit, in which case ions make small oscillations about a fixed point in the laboratory frame; in constrast, for the blobs considered here, the ions are frozen to the convecting blobs and do not make small oscillations in the laboratory frame. Also when one uses linear theory, the fluctuating potential is decomposed into its Fourier components, the motion is calculated due to each component, and the net motion is found by summing the motions due to each component. Here in contrast, the principle of superposition is invalid for the fluctuating potential, so one cannot calculate the motion by summing the contributions due to each Fourier component.

The characteristic distance between the peaks of two potential hills is $\sim 2 \pi / k_{\perp}$, so that at its base the hill radius is $\sim \pi / 2 k_{\perp}$. Hill vortices have the opposite sense of valley vortices. Ordinary ions (not suprathermal or stochastic) have $k_{\perp} r_{\mathrm{L}} \ll 1$ so that the gyroradius $r_{\mathrm{L}}$ is smaller than the blob size. Since ions go forever in the same closed vortex trajectory about their particular blob, the purely convective turbulent potential given by equation (3) cannot produce any ion transport.

\section{Orbits in finite-lifetime blobs}

Thus, if drift wave turbulence is going to cause ion transport, it will have to be because of absolute time variation, i.e. the second $t$ on the RHS of equation (2). Let us now allow such an absolute time variation and again change to the moving frame so that equation (2) becomes $\phi^{\prime}=\phi^{\prime}\left(x^{\prime}, y^{\prime}, t\right)$. The absolute time variation describes the random appearance and disappearance of blobs, plus random (i.e. not convective) velocities of blobs. If the blob time dependence is slow compared to the vortex orbit period, then, associated with the vortex orbit, there is an adiabatic invariant, the action $S=\oint \boldsymbol{P} \cdot \mathrm{d} \boldsymbol{l}$ where $\boldsymbol{P}=m \boldsymbol{u}_{\mathrm{E}}+q \boldsymbol{A}$ is the ion's canonical momentum and the line integral is one circuit around the vortex. Thus

$$
S=\oint\left(m u_{\mathrm{E}}+q \boldsymbol{A}\right) \cdot \mathrm{d} \boldsymbol{l}=m \oint \boldsymbol{u}_{\mathrm{E}} \cdot \mathrm{d} \boldsymbol{l}+q \Phi
$$

where $\Phi$ is the magentic flux enclosed by the vortex. The invariance of $S$ may be proven by realizing that if the potential has a slow time dependence, then the polarization drift acts to increase or decrease the area enclosed by the vortex orbit. Keeping only lowest-order terms, invariance is established by considering

$\frac{\mathrm{d} S}{\mathrm{~d} t} \approx m \int \frac{\mathrm{d} u_{\mathrm{E}}}{\mathrm{d} t} \cdot \mathrm{d} \boldsymbol{l}+q \cdot \frac{\mathrm{d}}{\mathrm{d} t} \int \mathrm{d} \boldsymbol{s}=\frac{m}{B^{2}} \int \frac{\mathrm{d} \boldsymbol{E}}{\mathrm{d} t} \times \boldsymbol{B} \cdot \mathrm{d} \boldsymbol{l}+q \boldsymbol{B} \cdot \oint \boldsymbol{u}_{\mathrm{P}} \times \mathrm{d} \boldsymbol{l}=0$

where $\mathrm{d} s$ is an element of the area enclosed by the vortex orbit. 
Let us now compare the two terms in $S$. To do this, consider the idealized hill blob of radius $\pi / 2 k_{\perp}$ which is azimuthally symmetric about its peak and which has the local potential $\phi^{\prime}\left(x^{\prime}, y^{\prime}, t\right)=\bar{\phi} f(t)\left[1-4 k_{\perp}^{2} r^{2} / \pi^{2}\right]$ where $r^{2}=\left(x^{\prime}-x_{0}\right)^{2}+\left(y^{\prime}-\right.$ $\left.y_{0}\right)^{2}$ and $x_{0}, y_{0}$ is the location of the peak of the hill. Defining the azimuthal angle around the hill to be $\theta$, gives $u_{\mathrm{E}}=-8 k_{\perp}^{2} r \bar{\phi} f(t) \hat{\theta} / B \pi^{2}$, so equation (5) becomes

$$
S=q B \pi r^{2}\left(1-16 \alpha f(t) / \pi^{2}\right) \text {. }
$$

We characterize the hill's life cycle by saying that initially $f\left(t_{0}\right)=1$ while at the hill's demise $f\left(\tau_{\text {life }}\right) \rightarrow 0$. If adiabatic invariance holds during the decay of the hill, then equating $S$ evaluated at $t_{0}$ and at $\tau_{\text {life, }}$ we find $r_{t=\tau_{\text {lffe }}^{2}}=r_{0}^{2}\left(1-16 \alpha / \pi^{2}\right)$. Because $\alpha \ll 1$ was assumed, $r$ is essentially constant during the decay and no ion transport occurs so long as $S$ remains an invariant.

Adiabatic invariance exists only for slowly changing periodic motions, i.e. only when $\omega_{\text {orbit }}^{-1} \mathrm{~d} \omega_{\text {orbit }} / \mathrm{d} t \ll \omega_{\text {orbit }}$ where $\omega_{\text {orbit }}$ is the angular frequency of the vortex orbit. Since $\omega_{\text {orbit }}=u_{\mathrm{E}} / r=8 k^{2} f(t) \bar{\phi} / B \pi^{2}$, adiabatic invariance exists when $\left|f^{-1} \mathrm{~d} f / \mathrm{d} t\right|<8 k_{\perp}^{2} \bar{\phi} f(t) / B \pi^{2}$, a condition clearly violated when $f \rightarrow 0$. Thus, adiabatic invariance is destroyed when the blob dies. If we assume that $f(t)=\exp \left(-t / \tau_{\text {life }}\right)$ then the condition for adiabatic invariance becomes $f>(\pi / 8 h) /\left[k_{\perp} L\left(e \bar{\phi} / T_{c}\right)\right] \approx 0.2$ using $h=2$ and equation (1). Hence, if equation (1) holds and the decay is exponential, adiabatic invarince exists until the blob amplitude decays to about one-fifth of its original peak value. The number of circumnavigations an ion makes in a blob lifetime is $N_{\text {orbit }}=u_{\mathrm{E}} \tau_{\text {lifc }} / 2 \pi r=\left(4 h / \pi^{2}\right) k_{\perp} L\left(e \bar{\phi} / T_{\mathrm{c}}\right) \sim 1$.

\section{Transport associated with finite-lifetime blobs}

An ion is consequently bound to a specific blob while the blob is alive, and no transport occurs. Only when the blob dies (i.e. $f<0.2$ ) is the adiabatic invariance destroyed and the ion released. After the blob dies, a new blob (hill or valley) will form and the ion will become attached to the new blob. The new blob will be on average a quarter wavelength away from the defunct blob and an average ion would have been located half way up/down the original blob and will be located half way up/down the new blob. The new blob will be oriented at an arbitrary angle in the $x-y$ plane relative to the $x$ axis, so that its rms angle relative to $x$ will be $\pi / 4$. Thus, the RMS step $\Delta x$ that occurs when an ion transfers its orbit from the old blob to the new blob will be $\Delta x \approx \lambda_{\perp} \cos (\pi / 4) / 4 \approx k_{\perp}^{-1}$. The time step associated with this step is the lifetime $\tau_{\text {life }} \approx h \pi / \omega^{*}$ of the original hill thus giving an $x$-direction diffusivity $D=(\Delta x)^{2} /$ time step $=\omega^{*} / \pi h k_{\perp}^{2}$. Using $k_{\perp} \rho_{\mathrm{s}} \approx 0.3$ and $\omega^{*} \approx k_{\perp} T_{\mathrm{c}} / e B L$ this becomes the gyro-Bohm diffusivity

$$
D \approx \frac{1}{h} \frac{\rho_{\mathrm{s}}}{L} \frac{T_{\mathrm{c}}}{e B}
$$

The main feature of this analysis is that it explicitly shows that in a collisionless plasma the discrete time steps required for random walk diffusion are produced by the abrupt breakdown of the adiabatic invariant $S$ which occurs when the blob amplitude decays to the point where $\omega_{\text {orbit }}^{-1} \mathrm{~d} \omega_{\text {orbit }} / \mathrm{d} t \sim \omega_{\text {orbit }}$.

Using for $T_{c}$ the mean temperature over the minor radius, i.e. $T_{c} \approx T_{c 0} / 2$, where $T_{\mathrm{e} 0}$ is the central (magnetic axis) temperature, equation (8) becomes

$$
D \approx 3.6 \times 10^{-5} T_{\mathrm{e} 0}^{3 / 2} \mu^{1 / 2} / B^{2} h L \mathrm{~m}^{2} \mathrm{~s}^{-1}
$$

where $T_{e 0}$ is in eV, $B$ is in $\mathrm{T}$, and $L$ is in $\mathrm{m}$, and $\mu$ is the ion mass in amu. 
The argument leading to equations (8) and (9) also shows why energetic ions are well confined. These ions have Larmor orbits much larger than the blob diameter (i.e. $k_{\perp} r_{1} \geqslant 1$ ). Unlike a low-energy ion which makes many cyclotron orbits while circumnavigating a blob, an energetic ion traverses many blobs during a single cyclotron orbit, so the effects of the individual blobs cancel.

It important to realize that blob lifetimes are related to the frequency bandwidth of the turbulence as observed in the wave frame but are not directly related to the frequency bandwidth as observed in the laboratory frame. This is because the turbulence typically has a large wavenumber bandwidth $\Delta k_{\perp}$ so that the frequency bandwidth observed in the laboratory frame will be $\sim u_{\mathrm{ph}}^{\mathrm{pl}} \Delta k_{\perp}$ which typically will be much larger than the intrinsic frequency bandwidth due to finite blob lifetime.

We have ignored ion motion along the parallel extent of the blob (which in three dimensions has a filamentary shape, with parallel half-wavelength $\sim \pi / k_{\|}$) because the ion parallel transit time $t_{\| \mathrm{i}}=\pi / k_{\| l} u_{\pi_{1}}$ is much longer than the vortex orbit period $t_{\text {orbit }}=\pi^{3} B / 4 k_{\perp}^{2} \bar{\phi}$. However, for electrons $\left.t_{\| \mathrm{e}} / t_{\text {orbit }}=\left(4 / \pi^{2}\right)\left(\omega / k_{\|} u_{\tau_{\mathrm{c}}}\right) k_{\perp} L\right)\left(e \bar{\phi} / T_{\mathrm{c}}\right)$. Since $e \bar{\phi} / T_{\mathrm{e}}$ is at best (Wooton et al 1990) a factor of 2 greater than $\tilde{n} / n=\left(k_{\perp} L\right)^{-1}$ in the interior, the electron parallel transit time is some moderate to large fraction of a vortex orbit period. Thus, the diffusion step size and time step for electrons should be comparable to the corresponding ion quantities.

If we assume that energy transport results from gyro-Bohm particle diffusion, then the energy confinement time will be $\tau_{\mathrm{E}} \approx a^{2} \kappa / 6 D$ where $k$ is the minor cross section vertical elongation. Assuming $L \sim a K^{1 / 2} / 2$ gives

$$
\tau_{\mathrm{E}} \approx 2.3 \times 10^{3} a^{3} B^{2} K^{3 / 2} h / T_{\mathrm{e} 0}^{3 / 2} \mu^{1 / 2} \mathrm{~s} .
$$

Equation (10) is just an example of the gyro-Bohm transport given by the Connor (1988) 'Model A' invariance scaling relation with index $q=-3 / 2$.

If temperature is eliminated in favour of power $P$ using the relation $n V T_{\mathrm{e} 0} / 2 \approx$ $P \tau_{E}$ where $V=2 \pi^{2} R a^{2}$ is the plasma volume, equation (10) becomes

$$
\tau_{\mathrm{E}} \approx 10^{2} R^{3 / 5} a^{12 / 5} n^{3 / 5} B^{4 / 5} K^{3 / 5} h^{2 / 5} P^{-3 / 5} \mu^{-1 / 5} \mathrm{~s} \text {. }
$$

Figure $1(b)$ lists $a, k, B$ and $T_{c 0}$, for a broad range of tokamak, stellarator, RFP. magnetic mirror and spheromak devices. The majority of the data in figure $1(b)$ are from the World Survey of Activities in Controlled Fusion Research (IAEA 1986); the rest is from Tomabechi (1990), Waltz et al (1990), JT-60 team (1990), Sigmar et al (1990) (tokamaks), Hirano et al (1990) and Almagri et al (1991) (RFPs), and Wysocki et al (1990) (spheromak). Figure $1(b)$ also plots the measured $\tau_{\mathrm{E}}$ versus the $\tau_{\mathrm{E}}$ predicted by equation $(10)$ for these devices. The additional unlabelled points comprise the measured and predicted $\tau_{E}$ of all significant devices of these types listed in the World Survey of Activities in Controlled Fusion Research. Unless the reference specified otherwise, $\mu=1$ was assumed, For RFPs the magnitude of $B$ is evaluated using the wall poloidal field $B_{\theta \text { wall }}=\mu_{0} I_{\mathrm{p}} / 2 \pi a$. Besides showing the well known result that equation (10) gives a reasonable fit to large tokamaks, figure $I(b)$ also shows that gyro-Bohm transport gives an upper bound for the energy transport of all significant magnetic confinement schemes attempted to date. This indicates that other (more deleterious) transport mechanisms dominate gyro-Bohm diffusion in some devices, but gyro-Bohm transport is always operative.

Also, using $h / \mu^{1 / 2}=1$, equation (10) is found to be consistent with the $\tau_{\mathrm{E}}\left(T_{\mathrm{e}}\right)$ dependence recently observed in TFTR (Efthimion et al 1991). In particular, equation (10) predicts $\tau_{\mathrm{E}}=0.23,0.14,0.11$ and $0.10 \mathrm{~s}$ versus the measured $\tau_{\mathrm{E}}$ of 
$0.27,0.127,0.105$ and $0.10 \mathrm{~s}$ for $T_{\mathrm{e} \beta}=2.3,3.6,4.1$ and $4.45 \mathrm{keV}$ respectively, with $a=0.93 \mathrm{~m}, B=4 \mathrm{~T}, k=1$.

\section{Application to H-mode}

To lowest order $S \approx \Phi$, so the area enclosed by each particle's vortex orbit is conserved, and since the blob is just the sum of its constituent particles, the blob area is a conserved quantity (so long its amplitude satisfies $f>0.2$ ). Associated with the $\mathrm{H}$ mode is a shear in the poloidal velocity, and so unlike equation (4) there is no blob rest frame. Blobs are stretched by the sheared velocity, but having constant area become elongated in the poloidal direction and thinner in the radial direction. This decreases the radial step size and so reduces radial diffusion. This velocityshear induced, time-dependent stretching of the blobs is analogous to the magneticshear induced, $z$-dependent stretching and twisting of static equipotential patterns experimentally observed by Mosher and Chen (1970).

\section{Stochasticity}

Let us now return to stochasticity. McChesney et al (1987) showed that orbits in a one-dimensional wave became stochastic when $\alpha>1$. Since $\alpha$ does not contain $\omega$, stochastic orbits can develop in a time-independent potential and since $\alpha \sim m$, stochastic orbits are important for ions, but not electrons. McChesney et al (1987) also showed that stochasticity corresponded to a breakdown of guiding centre theory, rendering meaningless the $\boldsymbol{E} \times \boldsymbol{B}$ and polarization drifts implicit in the adiabatic invariant $S$ of equation (5). Let us now instead rely on the more fundamental, but more restrictive, assumption of geometric symmetry to provide an ignorable coordinate. We consider the motion of an ion in the azimuthally symmetric, time-independent potential hill $\phi^{\prime}=\bar{\phi}\left(1-4 k_{\perp}^{2} r^{2} / \pi^{2}\right)$, where $r, \theta$ are defined as before. Because of the azimuthal symmetry the canonical angular momentum $P_{\theta}=m r^{2} \dot{\theta}+q r A_{\theta}$ is exactly conserved. Since $A_{\theta}=B r / 2$, we find $\dot{\theta}=\left(P_{\theta}-q B r^{2} / 2\right) / m r^{2}$ so the time-independent Hamiltonian becomes

$$
H=\frac{P_{r}^{2}}{2 m_{\mathrm{i}}}+\frac{\left(P_{\theta}-q B r^{2} / 2\right)^{2}}{2 m_{\mathrm{i}} r^{2}}+q \bar{\phi}\left(1-\frac{4 k_{\perp}^{2} r^{2}}{\pi^{2}}\right)
$$

Equation (13) describes particle motion in the one-dimensional effective potential

$$
V(r)=P_{\theta}^{2} / 2 m_{\mathrm{i}} r^{2}+\left[m_{\mathrm{i}}\left(\omega_{\mathrm{o}} / 2\right)^{2} r^{2} / 2\right]\left(1-32 \alpha / \pi^{2}\right) .
$$

When $\alpha<\pi^{2} / 32, V(r)$ has a minimum and ions circumnavigate the hill with some oscillation in $r$; this oscillation is just the $r$ component of the Larmor orbit. However, if $\alpha>\pi^{2} / 32$, i.e. $\left(k_{\perp} \rho_{s}\right)^{2}\left(e \bar{\phi} / T_{c}\right)>0.3$, then $V(r)$ monotonically decreases with $r$ and the ion orbit becomes unbound. The ion will then fall down the potential hill into the adjacent valley, gaining random kinetic energy since its direction is random. Since the distinction between stochastic and non-stochastic orbits is 
instantaneous, ions are quickly and locally heated as they are ejected from steep, tall hills (i.e. those satisfying $\left.\left(k_{\perp} \rho_{\mathrm{s}}\right)^{2}\left(e \bar{\phi} / T_{\mathrm{c}}\right)>0.3\right)$.

\section{Summary}

We have shown how breakdown of the adiabatic invariant $S$ at the end of the life of a blob results in gyro-Bohm diffusion. Gyro-Bohm diffusion has been shown to provide an upper bound for the transport in all significant magnetic confinement schemes, in addition to the large tokamaks to which it has traditionally been applied. If, as is usually the case, $e \tilde{\phi} / T_{e}>\tilde{n} / n \approx\left(k_{\perp} L\right)^{-1}$, this diffusion is not proportional to the turbulence amplitude and so should be important in the plasma interior even though interior turbulence levels are low $(\sim 1-5 \%)$. The key parameter is the blob lifetime $h$.

Consideration of ion orbits in steep $2 \mathrm{D}$ potential hills gives a stochasticity threshold of $\left(k_{\perp} \rho_{\mathrm{s}}\right)^{2}\left(e \bar{\phi} / T_{\mathrm{e}}\right)=0.3$, a factor of three lower than the one-dimensional in McChesney et al (1987). Since e $\tilde{\phi} / T_{e} \approx 0.5-0.8$ is observed in the edge, ions on edge potential hills having $k_{\perp} \rho_{\mathrm{s}} \sim 0.6-0.8$ will be stochastically heated; such steep hills can be expected to form a non-negligible part of the turbulent edge spectrum.

\section{Acknowledgments}

The author acknowledges useful discussions with $\mathrm{R}$ A Stern about the instantaneous character of stochasticity and with $G$ Hammett about gyro-Bohm diffusion. This work was supported by DOE Grant DE-FG03-86ER53232 and NSF Grant PHY-9114146.

\section{References}

Almagri A et al 1991 Proc. Physics of Alternative Magnetic Confinement Schemes (Varenna, 1990) (Bologna: Editrice Compositori) p 223 (see also Dimarco J 1991 Proc. Physics of Alternative Magnetic Confinement Schemes (Varenna, 1990) (Bologna: Editrice Compositori) p 683)

Christiansen J P, Cordey J G and Thomsen K 1990 Nucl. Fusion 301183

Connor J W 1988 Plasma Phys. Control. Fusion 30619

Efthimion P C et al 1991 Phys. Rev. Lett. 66421

Hirano Y et al 1990 Plasma Physics and Controlled Nuclear Fusion Research (Proc. 13th Int. Conf., Washington, $D C, 1990)$ vol 2 (Vienna: IAEA) p 717

Horton W 1990 Phys. Rep. 1921 (see especially p 142)

IAEA 1986 World Survey of Activities in Controlled Fusion Research (Nuclear Fusion special supplement) (Vienna: IAEA)

JT-60 team 1990 Plasma Physics and Controlled Nuclear Fusion Research (Proc. 13th Int. Conf., Washington, $D C, 1990$ ) vol 1 (Vienna: IAEA) p 53

Liewer P C 1985 Nucl. Fusion 25543

Liu J and Horton W $1986 \mathrm{~J}$. Plasma Phys. 361

Mazzucato E 1982 Phys. Rev. Lett. 481828 (cf figure 2)

McChesney, J M, Stern R A and Bellan P M 1987 Phys. Rev. Lett. 591436

Mosher D and Chen F F 1970 Phys, Fluids 131328

Ritz Ch P, Lin H, Rhodes T L and Wooton A J 1990 Phys. Rev. Lett. 652543

Ritz Ch P et al 1988 Rev. Sci. Instrum. 591739

Sigmar D J 1990 Plasma Physics and Controlled Nuclear Fusion Research (Proc. 13th Int. Conf., Washington, $D C, 1990$ ) (Vienna: IAEA) p 455 
Synakowski E J et al 1990 Phys. Rev. Lett. 652255

Tomabechi K 1990 Plasma Physics and Controlled Nuclear Fusion Research (Proc. 13th Int. Conf., Washington, DC, 1990) vol 3 (Vienna: IAEA) p 217 (see also Post D 1990 Plasma Physics and Controlled Nuclear Fusion Research (Proc. 13th Int. Conf., Washington, DC, 1990) vol 3 (Vienna: IAEA) p 239)

Waltz R E, Deboo J C and Rosenbluth M N 1990 Physics. Rev. Lett. 652390

Wooton A J et al 1990 Phys. Fluids B 22879

Wysocki F J, Fernandez J C, Henins I, Jarboe T R and Marklin G J 1990 Phys. Rev. Lett. 6540 Zweben S J 1985 Phys. Fluids 28974

Zweben S J 1990 Bull. Am. Phys. Soc. 352109

Zweben S J and Gould R W $1983 \mathrm{Nucl}$. Fusion 231625

1985 Nucl. Fusion 24171 